\title{
La maestría en Rehabilitación Neurológica, proyecto interinstitucional de formación interdisciplinaria en la prevención de alteraciones en el neurodesarrollo
}

Carmen Sánchez ${ }^{1}$

\section{Introducción}

La maestría en Rehabilitación Neurológica inició en 1975. El programa se diseñó con base en los planteamientos que sustentaron el sistema modular de la UAM-Xochimilco. En sus inicios y durante la coordinación de la doctora Carmen Sánchez (fundadora), se desarrolló un proyecto de investigación sobre la plasticidad cerebral con el apoyo del doctor Paul Bach y Ryta del Smith Kettlewell Institute of Visual Science de la Universidad de Berkeley de San Francisco (que se encontraba colaborando en la UAM), y otro proyecto sobre los sistemas de biorretroalimentación y alteraciones congénitas con el apoyo del doctor Víctor Alcaraz del Departamento de Investigaciones Cerebrales de la UNAM (Laboratorio de Psicofisiología de la Facultad de Psicología). A partir de la segunda generación se integraron alumnos de diversas disciplinas (médicos, psicólogos y otras profesiones afines al campo) y se ampliaron las posibilidades de desarrollo del

1 Universidad Autónoma Metropolitana-X. 
posgrado con el abordaje preventivo de los factores de riesgo de secuelas neurológicas, que abrió la actual vertiente del neurodesarrollo en las líneas de investigación del posgrado.

\section{El Plan de Estudios Modular}

Los seis módulos y sus unidades de actividad instrumental que comprenden el plan de estudios se estructuraron integrando la docencia, investigación y servicio en torno a objetos problematizados o problemas concretos de la realidad, representados por el abordaje preventivo de las secuelas del desarrollo generadas por diversos daños al sistema nervioso en las etapas tempranas de la vida. La actividad del docente se diseñó para organizar el conocimiento y la articulación de contenidos y metodologías con la finalidad de permitirle al alumno adquirir y sistematizar el conocimiento existente y coordinar el conjunto de acciones y operaciones por realizar, como partícipe y generador de su propio proceso formativo a través de la investigación y el servicio. El servicio se ha considerado como el puente de vinculación de la universidad con los problemas de la sociedad y espacio privilegiado para favorecer la apropiación, generación y aplicación del conocimiento orientado a la solución de problemas socialmente relevantes. Esta propuesta es coincidente y contemporánea con la metodología educativa que se conoce como aprendizaje-servicio, concepto que refiere una propuesta educativa que combina procesos de aprendizaje y de servicio a la comunidad en un solo proyecto articulado, en el que los estudiantes se forman al trabajar sobre necesidades reales del entorno con el objetivo de mejorarlo (Puig y Palos, 2006). El aprendizaje-servicio ha sido implementado como estrategia metodológica en diversas universidades (Alonso, Arandia, Martínez, Martínez y Gezuraga, 2013; Martínez, Martínez, Alonso y Gezuraga, 2013; Rodríguez, 2014; Rubio, Prats y Gómez, 2013).

El servicio posibilita la aplicación del conocimiento adquirido durante la actividad de docencia y su generación a través de los proyectos de investigación o verificación empírica en casos 
concretos, considerando diversas teorías y metodologías de abordaje. Esto ha requerido como estrategia procedimental que el programa de la maestría se inserte en áreas de servicio diseñadas específicamente para generar y replicar el conocimiento, estrategia que se ha logrado a través del Laboratorio de Seguimiento del Neurodesarrollo, producto de un convenio interinstitucional entre el Instituto Nacional de Pediatría y la Universidad, cuyos objetivos son la investigación y la enseñanza a través del servicio.

\section{Sustento epistemológico}

Transformar el perfil profesional como práctica social en el desarrollo y aplicación del conocimiento generó la necesaria redefinición de las profesiones e inclusive el surgimiento de nuevas prácticas profesionales denominadas prácticas emergentes. Fue en este contexto donde surgió la necesidad de proponer un cambio en las prácticas hasta ese momento desarrolladas en el campo de la rehabilitación (modelo caracterizado por fragmentar la atención dependiendo del área afectada y considerando como eje rector de las acciones la secuela estructurada fundamentalmente de tipo motor y mental). Al cuestionar el enfoque tradicional de la rehabilitación, la maestría se propuso reflexionar sobre el concepto de discapacidad. En primer lugar, considerando al ser humano como la totalidad relacional que lo caracteriza (biológico-histórico-social), no puede rehabilitarse por partes. "La totalidad no es un agregado de elementos independientes, los elementos están sometidos a leyes de composición que confieren al todo propiedades distintas de la de sus elementos" (Piaget, 1997:142). Afirmar que la actividad de una totalidad no se explica en función de las partes permitió sustentar la necesidad de orientar las acciones a la totalidad y no únicamente en forma aislada a áreas afectadas o secuelas. Por otro lado, la rehabilitación tradicional al abordar la secuela como estado y determinante causal de la discapacidad ignoró el proceso que lleva a un sujeto a su estructuración, además de incidir en un momento tardío en su atención. La maestría planteó una necesaria redefi- 
nición, considerando que secuela y discapacidad son en su génesis dos momentos diferenciales en el proceso de desarrollo de un sujeto (la secuela considerada como condición necesaria pero no suficiente en la estructuración de la discapacidad); la secuela definida como la alteración irreversible en la estructura biológica del individuo independientemente de sus causas y consecuencias, mientras que la discapacidad como proceso no se genera a partir de la consolidación del daño (secuela), sino durante el proceso de morbilidad neurológica desencadenante del daño. En este sentido, es posible observar que previo a la estructuración de la secuela se manifiestan una serie de signos anormales de daño como son la presencia de reflejos o conductas aberrantes o la ausencia y/o persistencia de reflejos o conductas propias del desarrollo. Estos signos clínicos organizados como factores de riesgo para la estructuración de secuelas neurológicas conformarán un proceso sistemático de alteración del curso del desarrollo normal de un niño y dependiendo de las condiciones favorables o desfavorables del contexto histórico-social en el que se desenvuelve determinarán que un proceso de discapacidad se estructure. La discapacidad como proceso se manifestará cuando la función alterada implique limitaciones en la actividad y participación social de un sujeto en contextos socioculturales concretos, relación que sustenta momentos diferenciales para incidir en el proceso y en la solución del problema a través de ejercer acciones que permitan incidir en una etapa de prevención y no una vez que la secuela ha sido estructurada.

Estos elementos han permitido constituir una práctica unificadora, donde participan una diversidad de disciplinas en áreas especializadas de la salud y la educación, que desde perspectivas diversas del saber se articulan en la construcción de un campo profesional con carácter interdisciplinario para la atención preventiva e integral de las alteraciones del desarrollo infantil originadas por el daño al sistema nervioso central en edades tempranas, rompiendo con la tradicional formación profesional unidisciplinaria.

En cada uno de los módulos, la selección de los contenidos se ha orientado al análisis sobre la génesis del daño y los 
mecanismos de reparación y reorganización nerviosa, así como de los diversos enfoques paradigmáticos sobre los determinantes causales (biológicos, psicológicos y sociales) del proceso de desarrollo ante la normalidad como ante el daño, considerando las aportaciones de la epistemología genética como uno de sus principales ejes, donde el concepto de epigénesis ha mostrado su relevancia para explicar los procesos de transformación del desarrollo desde sus expresiones más primitivas y reflejas, hasta las construcciones de mayor complejidad del pensamiento.

\section{Interdisciplina y complejidad}

El principio de articulación del proceso educativo fue considerar que el abordaje de un objeto de estudio por su complejidad requiere necesariamente una aproximación interdisciplinaria para lograr el objetivo de conocer y transformar la realidad. La complejidad del problema de la discapacidad con relación a sus causas y consecuencias emergen de su carácter multidimensional y multicausal de los elementos o variables que lo constituyen como proceso. Su ámbito de repercusión en el campo de la salud no puede restringirse a lo individual ni a la estructura biológica, sino a la relación dinámica que existe entre lo individual y lo colectivo, lo biológico y lo social. La comprensión de este complejo proceso de interrelación no puede ser posible por ninguno de sus elementos en particular, sino en función de la articulación de éstos en el todo (daño orgánico, susceptibilidad y capacidad reorganizativa de las estructuras biológicas del individuo ante el daño, severidad de las expresiones clínicas, diversidad de contextos sociales y culturales).

Las visiones unidisciplinarias han restringido desde su óptica particular la explicación de los factores que intervienen en el problema, limitando las acciones para su solución. Éste es el caso de prácticas médicas que han centrado la acción de rehabilitación en el manejo fisioterapéutico de las secuelas estructuradas, así como prácticas pedagógicas centradas en el logro de objetivos de aprendizaje al margen tanto de las manifestaciones 
clínicas del daño como de las alteraciones en el desarrollo. Éstas representan los obstáculos de mayor trascendencia para el logro del proceso educativo y de la integración familiar, escolar y social de los niños.

El trabajo interdisciplinario se ha favorecido al considerar que la rehabilitación como práctica individual y social representa un campo que requiere la articulación de diferentes disciplinas involucradas en la comprensión y solución de los problemas que genera el daño neurológico en el desarrollo integral de un individuo. Un mismo objeto de estudio puede ser abordado desde diferentes disciplinas. La posibilidad de trabajo interdisciplinario podría considerarse cuando diversos campos disciplinarios coinciden en un objeto de conocimiento común. Considerar como objeto de estudio común el daño estructural y funcional de uno de los sistemas de mayor complejidad en la organización ontogenética, así como sus repercusiones en el proceso de desarrollo del individuo en los diversos contextos de su integración social, por la complejidad que representa la cantidad de elementos interrelacionados, trasciende los límites de una disciplina y una metodología de estudio, requiriendo como objeto de conocimiento un abordaje amplio desde diversos campos de las ciencias.

\section{Referencias}

Alonso, S.I., Arandia, L.M., Martínez, D.I., Martínez, D.B. y Gezuraga, A.M. (2013). El Aprendizaje-Servicio en la innovación universitaria. Una experiencia realizada en la formación de educadoras y educadores sociales. Revista Internacional para la Justicia Social, 2(2), 195-216.

Martínez, D.B., Martínez, D.I., Alonso, S.I. y Gezuraga, A.M. (2013). El aprendizaje-servicio, una oportunidad para avanzar en la innovación educativa dentro de la Universidad del País Vasco. Tendencias Pedagógicas, 21, 99117.

Piaget, J. (1997). Biología y conocimiento (12ª edición). México: Siglo XXI. 
Plan y Programas de Estudio de la Maestría en Rehabilitación Neurológica. (2006). México, D.F.: UAM-X. Documento interno.

Puig, J. y Palos, J. (2006). Rasgos pedagógicos del aprendizaje-servicio. Cuadernos de Pedagogía, 357, 60-63. Recuperado de http://www.europa.sim.ucm.es.

Rodríguez, G.M. (2014). El Aprendizaje-Servicio como estrategia metodológica en la universidad. Revista Complutense de Educación, 25(1), 95-113.

Rubio, L., Prats, E. y Gómez, L. (2013). Universidad y sociedad. Experiencias de aprendizaje servicio en la universidad. Colección Educación y Comunidad, 8. Barcelona: ICE-UB. 
